\title{
Conceptual Design of the Fermilab Nb3Sn High Field Dipole Model
}

\author{
G. Ambrosio, N. Andreev, T. Arkan, E. Barzi, D. Chichili, V. Kashikhin, P.J. Limon, \\ T. Ogitsu, J.Ozelis, I. Terechkine, J.C. Tompkins, S. Yadav, R. Yamada, \\ V. Yarba, A.V. Zlobin*, FNAL, Batavia, IL, USA \\ S. Caspi, LBNL, Berkeley, CA, USA, \\ M. Wake, KEK, Tsukuba, Japan
}

\begin{abstract}
A short dipole model with the 10-11 T nominal field based on the $\mathrm{Nb} 3 \mathrm{Sn}$ superconducting strand is being developed at Fermilab in collaboration with LBNL and KEK as part of the R\&D effort for VLHC. This paper describes the magnet conceptual design and parameters as well as the results of magnetic field, mechanical and quench protection calculations. Parameters of the superconducting strand and cable are also reported.
\end{abstract}

\section{INTRODUCTION}

Among the approaches for a post-LHC Very Large Hadron Collider (VLHC) of $100 \mathrm{TeV}$ center-of-mass energy are designs based on superconducting High-Field Magnets (HFM). A VLHC built with HFM has the advantage of relatively small machine circumference and emittance damping due to synchrotron radiation, but has to accommodate the radiation power absorbed in the beam tube. While an optimum field range has yet to be determined, an operational field between $10 \mathrm{~T}$ and $12 \mathrm{~T}$ results in sufficient radiation damping effect without overpowering and significant complication the cryogenic and vacuum systems of the machine.

Development of HFM with the 10-12 T nominal field based on the $\mathrm{NbTi}$ strand is practically impossible because of the upper critical field limitation. Alternative superconductors for high field magnets are A15 alloys and $\mathrm{Nb3Sn}$ in particular, having higher critical magnetic field and critical temperature. Recent progress in the development of $\mathrm{Nb} 3 \mathrm{Sn}$ superconducting strands [1] makes it possible to design cost-effective accelerator magnets with a nominal field range of 10-12 $\mathrm{T}$ based on a shell-type $(\cos \theta)$ coil geometry. Extensive studies of shell-type designs with small bore diameter of $30-50 \mathrm{~mm}$, with a different current block arrangement and cable parameters, etc., were carried out during last year. Their main goal was the determination of optimal magnet parameters, development of the robust and cost effective design and technology. The results of these studies will be reported separetelly. This paper presents the description of the conceptual design of the first Nb3Sn short models to be fabricated and tested at Fermilab in frame of High Field Magnet R\&D program.

Work supported by the U.S. Department of Energy

*E-mail: zlobin@fnal.gov

\section{MAGNET DESIGN}

The design of the HFM consists of two layer shelltype coil with a $44.5 \mathrm{~mm}$ bore and cold iron yoke. Figure 1 shows the magnet coil cross-section.

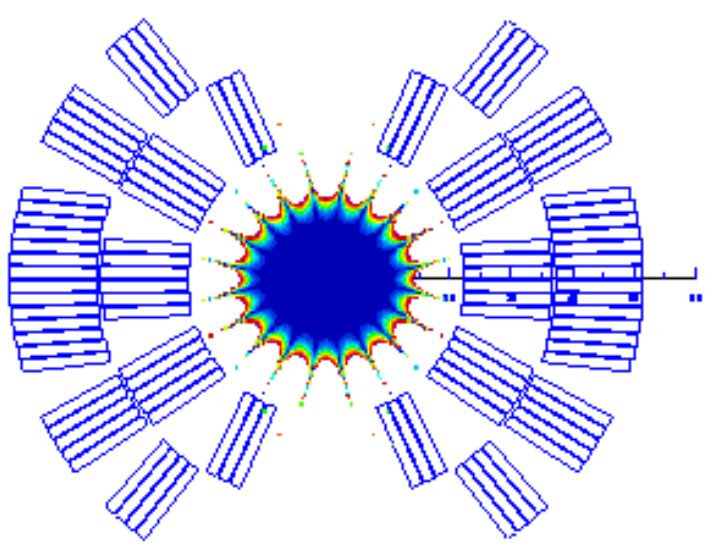

Figure 1: HFM coil cross-section.

One keystoned Rutherford-type Nb3Sn cable is used in both inner and outer layer. The cable has the width of $14.24 \mathrm{~mm}$, the mid-thickness of $1.80 \mathrm{~mm}$ and the keystone angle of $\sim 1$ degree. Strand and cable parameters are presented in Table 3 and 4 . The cable is insulated with a high temperature ceramic insulation having $125 \mu \mathrm{m}$ azimuthal and $125 \mu \mathrm{m}$ radial thickness [2].

Each coil consists of 27 turns, 11 turns in the inner layer and 16 turns in the outer layer. The inter-layer insulation thickness is $0.28 \mathrm{~mm}$ and the thickness of the mid-plane insulation is $2 \times 0.125 \mathrm{~mm}$ for each layer. Each coil has four spacers per quadrant, two for each layer, which are used to minimize the low order geometrical harmonics and to ensure the radial turn position in the coil. The coil ends have also a blockwise layout of turns to reduce the maximum field and to improve the end field quality.

The iron yoke has an inner diameter of $120 \mathrm{~mm}$ and an outer diameter of $400 \mathrm{~mm}$. The yoke inner diameter was determined by a chosen thickness of the coil/yoke spacer. The iron outer diameter was chosen based on the fringe field reduction, minimization of yoke volume and available contact tooling. No special holes are used at 
this stage. The final optimization of the iron yoke crosssection will be done later.

Calculated magnet design parameters are presented in Table 1.

Table 1: Magnet design parameters

\begin{tabular}{|l|c|c|}
\hline \multicolumn{1}{|c|}{ Parameter } & Unit & Value \\
\hline Magnet bore diameter & $\mathrm{mm}$ & 44.5 \\
\hline Maximum central field & $\mathrm{T}$ & 12.28 \\
\hline Short sample limit & $\mathrm{kA}$ & 18.14 \\
\hline Central field transfer function & $\mathrm{T} / \mathrm{kA}$ & 0.7407 \\
\hline Coil current @ 11 T central field & $\mathrm{kA}$ & 16.25 \\
\hline Stored energy @ 11 T & $\mathrm{kJ} / \mathrm{m}$ & 252 \\
\hline Magnet inductance & $\mathrm{mH} / \mathrm{m}$ & 1.91 \\
\hline Coil area & $\mathrm{mm}$ & 2512 \\
\hline
\end{tabular}

The maximum central field presented in Table 1 was calculated without the iron saturation effect and cable degradation effect. These effects reduce the maximum field by $5-10 \%$.

The design was optimized to increase the inner coil pole width and to reduce the coil volume. The large pole width of $16.36 \mathrm{~mm}$ in this design is quite comfortable for the inner-layer pole-turn winding. The coil cross-section area in this design is by factor of 1.9 smaller than one in MSUT (design field is $11.4 \mathrm{~T}$ ) [3] and by factor of 2.7 smaller than D20 coil cross-section (design field is $13 \mathrm{~T}$ ) [4].

The design low-order harmonics for the dipole central field and harmonics RMS spread for block random displacements within $\pm 50 \mathrm{um}$, calculated with ROXIE [5] assuming an iron yoke permeability of 1000, are reported in Table 2 at $1 \mathrm{~cm}$ bore radius.

Table 2: Field harmonics

\begin{tabular}{|c|c|c|}
\hline $\mathrm{n}$ & bn & $\sigma(\mathrm{an}), \sigma(\mathrm{bn})$ \\
\hline 2 & - & 1.198 \\
\hline 3 & -0.000 & 0.564 \\
\hline 4 & - & 0.279 \\
\hline 5 & 0.000 & 0.103 \\
\hline 6 & - & 0.047 \\
\hline 7 & -0.007 & 0.021 \\
\hline 8 & - & 0.008 \\
\hline 9 & -0.071 & 0.005 \\
\hline 10 & - & 0.001 \\
\hline 11 & 0.103 & 0.003 \\
\hline
\end{tabular}

Both geometrical (systematic) low order harmonics and their expected RMS spread are small and meet the field quality requirements for the accelerator magnets [6].

$\mathrm{Nb} 3 \mathrm{Sn}$ strands produced using the internal tin process have quite high effective filament diameter of $\sim 70-100$ um. Expected contribution of coil magnetization for $\mathrm{d}_{\text {eff }}=70 \mu \mathrm{m}$ to b3 is -40 units and to b5 is +5 units at $1 \mathrm{kA}$. These values are too large and must be significantly reduced by reducing the effective filament diameter to the level less than 10-20 $\mu \mathrm{m}$. Eddy current magnetization effects, related to the eddy currents in strands and in the cable, are not presented here. They will be reduced using small filament twist pitch and providing a high interstrand resistance in the cable.

The effect of iron saturation on the field quality is also quite large. The range of variation for $b_{3}$ is within 6-7 units at $1 \mathrm{~cm}$ reference radius for the chosen yoke crosssection. The effect on higher order harmonics is small. Iron saturation effect will be corrected in future by optimizing the hole size and position in iron yoke.

Magnet protection in case of quench provided by internal quench heaters. They are installed in between the inner and outer layers to quench all inner and outer turns. Quench analysis was done for one operating heater.

According the calculations to keep the cable maximum temperature after quench below $300 \mathrm{~K}$, the quench integral has to be less than $16 \cdot 10^{6} \mathrm{~A}^{2} \mathrm{~s}$. At current of $16 \mathrm{kA}$ the heater induced quench integral is $11 \cdot 10^{6} \mathrm{~A}^{2} \mathrm{~s}$. It corresponds to the coil temperature under heater $160 \mathrm{~K}$ in the inner layer and $120 \mathrm{~K}$ in the outer layer. The calculated maximum turn-to-turn voltage is less than $15 \mathrm{~V}$ and coil-ground voltage does not exceed $100 \mathrm{~V}$ for $1 \mathrm{~m}$ long model. As it can be seen, the quench protection scheme with inter-layer heaters provides low maximum coil temperature and low voltages in the magnet.

\section{COIL SUPPORT STRUCTURE}

HFM cold mass cross-section is shown in Figure 2. The coils are mechanically supported by the vertically split iron laminations locked with two aluminum or stainless steel clamps and the stainless steel helium vessel skin. Thick end plates are used to restrict the longitudinal coil motion under Lorentz forces.

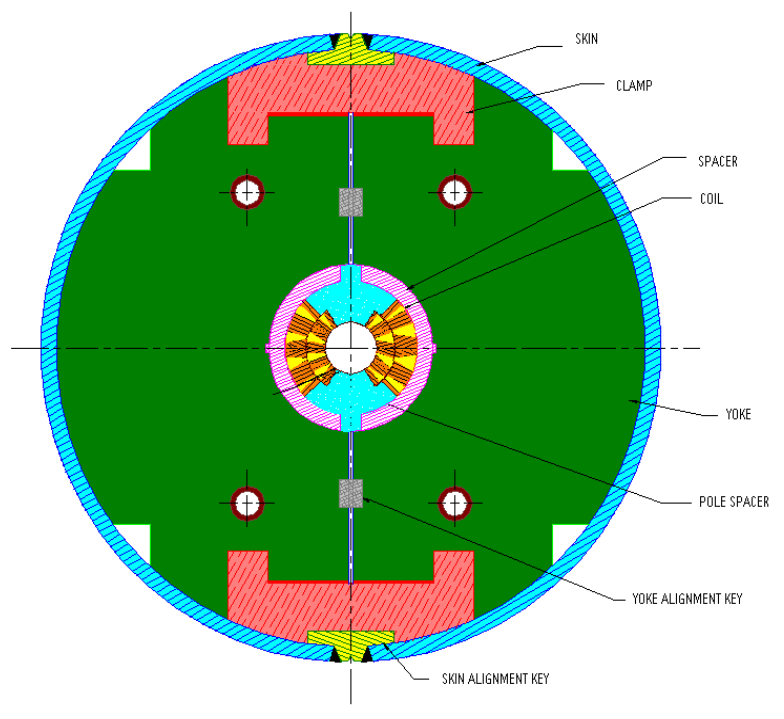

Figure 2: Cold mass cross-section.

Two $8 \mathrm{~mm}$ thick aluminum or brass spacers fill the space between the coil and yoke and protect the coil 
during assembling. The initial coil preload during assembling and the magnet geometry (vertical gap) control at room temperature is provided by two clamps. Final coil prestress at operation temperature, necessary to reduce the radial and azimuthal turn motion under Lorentz force action, is created with the help of a two-piece iron yoke, two clamps and a welded stainless steel helium vessel skin.

Preliminary calculations show that maximum radial and azimuthal stresses applied to the cable in the coil is less than $100 \mathrm{MPa}$. Finite element analysis using ANSYS is being performed now to optimize the azimuthal coil prestress at room and at operation temperatures and to determine tolerances and maximum stresses in the major elements of magnet support structure.

\section{CONDUCTOR DEVELOPMENT}

The superconducting strands for the HFM first short model are being produced by IGC using internal tin process. Strand parameters are summarized in Table 2.

Table 2: Nb3Sn strand parameters

\begin{tabular}{|l|c|c|}
\hline \multicolumn{1}{|c|}{ Parameter } & Unit & Value \\
\hline Strand diameter & $\mathrm{mm}$ & 1.00 \\
\hline Effective filament diameter & $\mu \mathrm{m}$ & $<70$ \\
\hline Cu:nonCu ratio & & $0.85: 1$ \\
\hline Residual resistivity ratio (RRR) & & $>75$ \\
\hline Twist pitch & $\mathrm{mm}$ & 15 \\
\hline Ic(12T,4.2K) & $\mathrm{A}$ & 800 \\
\hline Jc $(12 \mathrm{~T}, 4.2 \mathrm{~K})$ & $\mathrm{kA} / \mathrm{mm}^{2}$ & 1.886 \\
\hline
\end{tabular}

The R\&D program aimed to improve the Nb3Sn strand parameters and to reduce its cost supported by U.S. Labs, universities and industry has been started. The main goal of the program is to increase a critical current density $\mathrm{J}_{\mathrm{C}}(12 \mathrm{~T}, 4.2 \mathrm{~K})$ in strands up to $2000-2500 \mathrm{kA} / \mathrm{mm}^{2}$ at an effective filament diameter of 20 um or less.

The main HFM cable parameters are listed in Table 3.

Table 3: Cable parameters

\begin{tabular}{|l|c|c|}
\hline \multicolumn{1}{|c|}{ Parameter } & Unit & Value \\
\hline Number of strands & & 28 \\
\hline Strand diameter & $\mathrm{mm}$ & 1.00 \\
\hline Cable width & $\mathrm{mm}$ & 14.24 \\
\hline Mean thickness & $\mathrm{mm}$ & 1.800 \\
\hline Minor edge & $\mathrm{mm}$ & 1.687 \\
\hline Major edge & $\mathrm{mm}$ & 1.913 \\
\hline Packing factor & & 0.884 \\
\hline
\end{tabular}

Cable samples with different packing factor have been fabricated by LBNL and tested at Fermilab. Critical current degradation with respect to round strand measured on the strands extracted from cable as function of the cable packing factor is shown in Figure 2.

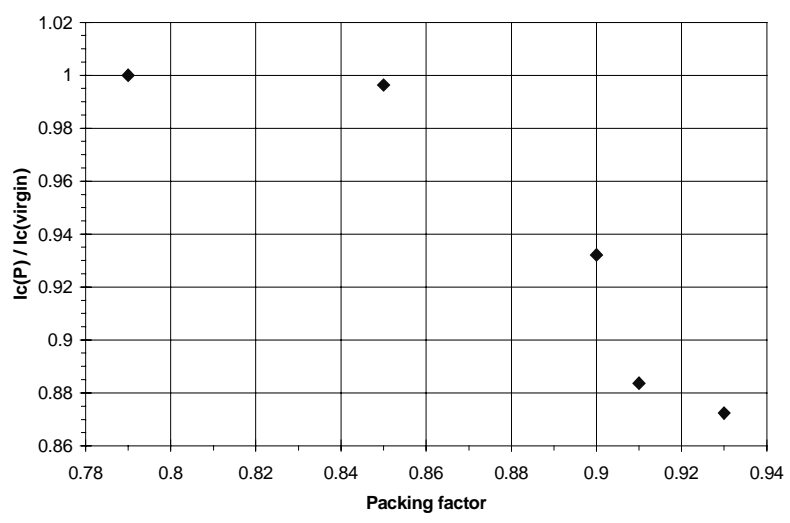

Figure 2: Effect of packing factor on the cable critical current degradation.

The data show that the critical current degradation after cabling for $\mathrm{Nb} 3 \mathrm{Sn}$ strand could be less than $5 \%$ if cable packing factor does not exceed $88 \%$. Based on the results of stress calculations the additional cable critical current degradation caused by the azimuthal and radial stresses applied to the cable in the coil at expected stress level will be reversible and small, less than 6\% [7].

\section{CONCLUSIONS}

The design of Nb3Sn dipole model, developed by the Fermilab/LBNL/KEK collaboration, allows to reach the maximum field of 11-12 $\mathrm{T}$ with existing Nb3Sn superconducting strand and to reduce signifficantly the coil cross-section. The presented design concept as well as the basic technological solutions will be studied experimentally on a series of short models. The development of HFM engineering design and tooling has been started. Tests of the first short model are planned for the Fall of 2000.

\section{REFERENCES}

[1]. E. Gregory and T. Pyon, "Some Recent Developments In Low Temperature Superconductors For Fusion, High Energy Physics And Other Applications", Proc. of ICEC'17, Bournemouth (UK), 1998, p.399.

[2]. C. Chichili et al., "Niobium-Tin Magnet Technology Development at Fermilab ", presented at PAC'99.

[3]. A. den Ouden et al., "Application of Nb3Sn Superconductors in High-Field Accelerator Magnets", IEEE Trans. on Applied Superconductivity, Vol. 7, No. 2, June 1997, p.733.

[4]. A.D. McInturff et al., "Test Results for a High Field (13 T) Nb3Sn Dipole", PAC'97

[5]. S. Russenschuck, "A Computer Program for the Design of Superconducting Accelerator Magnets", CERN AC/95-05 (MA), September 1995.

[6]. Superconducting Super Collider, Conceptual Design, SSC-SR2020, March 1986, p.151

[7]. H. ten Kate, "Recent Developments on PIT Nb3Sn conductors", High Field Accelerator Magnet Workshop, Erice, March 12, 1999. 